\title{
Plantar plate pathology: a review article
}

\begin{abstract}
The plantar plate is a thick fibrocartilage tissue located at the ball of the foot. Pathology of the plantar plate is frequently missed or misdiagnosed and additionally, can be challenging to treat. Current surgical techniques include direct and indirect repairs. The purpose of this article is to help better understand and review the evaluation and treatment of plantar plate tears.
\end{abstract}

Keywords: Plantar plate, lesser metatarsophalangeal joint, Weil metatarsal osteotomy, Lachman drawer, Kelikian pushup test

\author{
Volume 6 Issue 6 - 2016
}

Michael Maier, Peter Pham,Alexis Kreplick, Michael Ringwood

Texas Sports Medicine Institute, USA

Correspondence: Michael Maier, Texas Sports Medicine Institute, 7830 W, Grand Parkway S, Suite 280 Richmond,TX 77406, USA, Tel 28I-633-4940 Email mmaiermd@gmail.com

Received: October 05, 2016 | Published: December 14, 2016

\section{Introduction}

Plantar plate pathology including inflammation, attenuation, and rupture are common sources of forefoot pain and deformity. First discussed in the $1980 \mathrm{~s}$, numerous terms are used to describe this condition including metatarsalgia, pre-dislocation syndrome, lesser metatarsophalangeal joint (MTPJ) instability, and crossover toe deformity. ${ }^{1}$ It is important to recognize that these diagnoses are common manifestations of plantar plate dysfunction.

The second MTPJ is the most frequently affected with the incidence of this condition highest among women over the age of $50 .^{2}$ Interestingly, patients with rheumatoid arthritis tend to demonstrate plantar plate insufficiency at the fourth and fifth MTPJs more so than the second. ${ }^{3}$ Other deformities are often present in conjunction such as hallux abducto valgus (Figure 1), hallux rigidus, and hallux varus. ${ }^{4}$ This review will focus on plantar plate anatomy, pathology, and the diagnosis, treatment and outcomes of plantar plate tears.

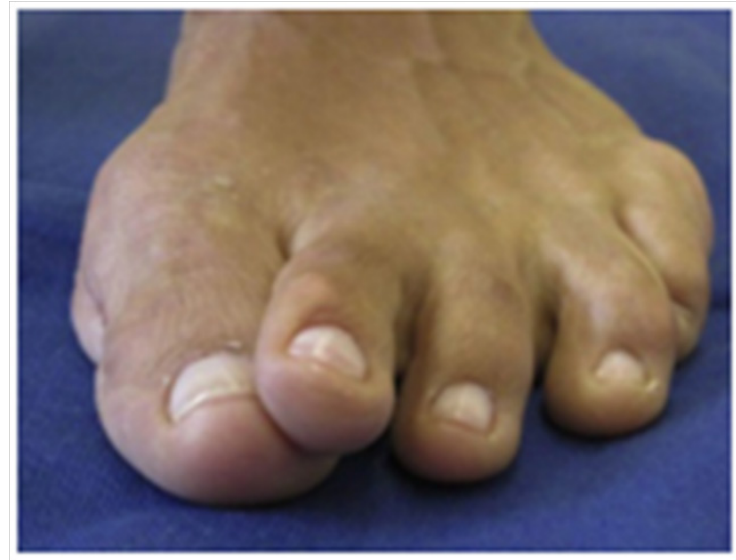

Figure I Typical appearance of a ruptured plantar plate with concomitant hallux abducto valgus deformity. Note the dorsal dislocation of the digit with a medial. ${ }^{18}$

\section{Anatomy}

The plantar plate is a fibrocartilaginous, intracapsular structure composed of primarily type 1 collagen measuring approximately $20 \mathrm{~mm}, 16 \mathrm{~mm}$, and $2-5 \mathrm{~mm}$ in length, width, and thickness, respectively. The plantar plate inserts firmly to the bases of the proximal phalanges (Figure 2) and serves as an important distal attachment of the plantar fascia. ${ }^{6}$ It is anchored to the distal metaphyseal region of the lesser metatarsals by the collateral ligaments and each plantar plate apparatus is coupled together by the deep transverse metatarsal ligament. ${ }^{7}$ The plantar plate is in direct contact with the lesser metatarsal heads during gait and functions as the primary static stabilizer of the lesser MTPJs. ${ }^{8}$ The structure and function is analogous to the menisci of the knee by resisting compressive forces during weight bearing. ${ }^{5}$

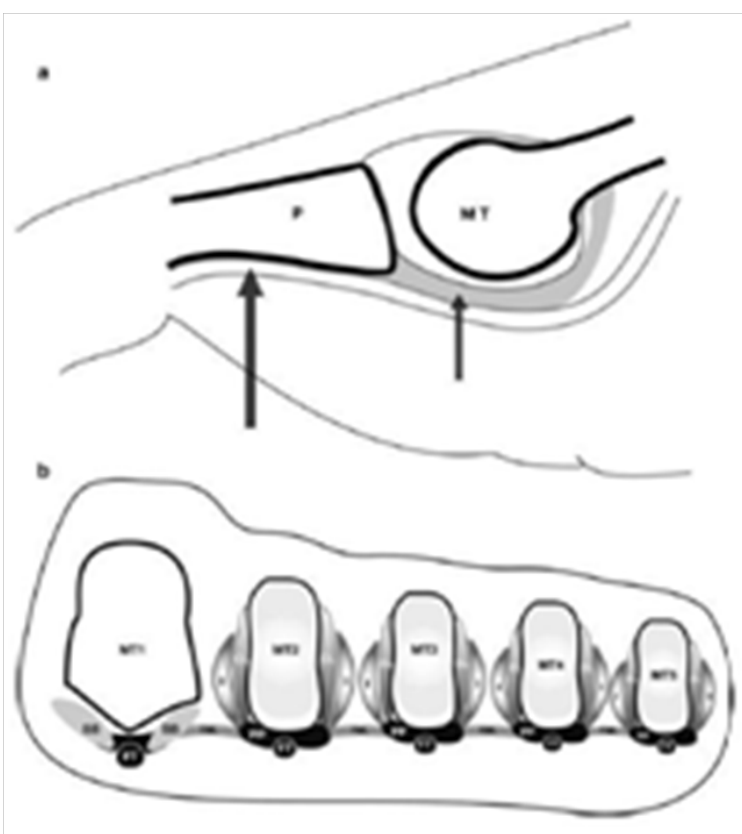

Figure 2a Sagittal view of a lesser MTPJ demonstrating the insertion of the plantar plate (short arrow) into the base of the proximal phalanx $(P)$ with the flexor tendon sheath (long arrow) immediately plantar.

Figure $\mathbf{2 b}$ Coronal view through the forefoot.

The plantar plate is closely associated with theinterosseous muscles (I), the flexor tendon sheath ( FT) and the deep transverse metatarsalligament ( TML). ${ }^{19}$

\section{Pathophysiology}

The etiology of plantar plate pathology is multifactorial. ${ }^{9-12}$ Any biomechanical abnormality leading to overload of the lesser metatarsals may result in plantar plate deterioration and subsequent MTPJ instability; this includes a long second metatarsal, first ray hypermobility, hallux abducto valgus, hallux rigidus, and pes planus. ${ }^{4,9}$

When the plantar plate ruptures and dorsal displacement of the proximal phalanx occurs, the ability of the intrinsic and extrinsic 
musculature to provide dynamic stability of the lesser MTPJs is impaired. Changes of the biomechanical axes of the primary flexors of the lesser MTPJs (the interosseous muscles) in addition to the lumbricals, flexor digitorum brevis, and flexor digitorum longus result in a defective restraint to prevent dorsal subluxation of the toe. ${ }^{10}$ The chronic inflammatory state of synovial tissues in rheumatoid arthritis and other systemic arthritic diseases causes soft tissue and bone destruction which progresses to joint insufficiency and often frank dislocation of the lesser MTPJs. ${ }^{11}$ Less commonly, acute trauma produces this condition. ${ }^{12}$

\section{Diagnosis}

Physical examination is paramount in the diagnosis of plantar plate dysfunction. Forefoot pain and specifically discomfort involving the metatarsal heads can arise from a variety of pathologies. During physical examination, a practitioner should employ dislocatable and muscular tests, radiographic imaging, and on occasion, advanced imaging such as MRI.

Plantar plate dysfunction can occur as a chronic, gradual disease process or more infrequently as an acute injury. Physical examination throughout the advancement of plantar plate disease changes drastically. Initially, patients may present with plantar sulcus pain, swelling at the MTPJ and no noticeable digital deformity. With time, digital dislocation, increasing deformity and intensifying pain characterize later stages. ${ }^{4,9}$ A practitioner can use multiple exams to elucidate the diagnosis of plantar plate injury. A Kelikian pushup test, manual loading of plantar plate by applying direct pressure upon the metatarsal heads, should be performed and reveal reduction to the level of surrounding digits in a patient without plantar plate pathology. The Lachman drawer maneuver (Figure 3a) consists of applying vertical stress to the proximal phalanx with one hand while stabilizing the metatarsal of the involved MTPJ with the other hand; greater than $2 \mathrm{~mm}$ of dorsal displacement is significant. Additionally, the paper pull-out test, in which a thin strip of paper is placed beneath the pulp of the toe with the toe in plantarflexion. A positive test will reveal decreased strength in plantarflexion of the involved digit, resulting in a completely intact piece of paper pulled from beneath the digit. ${ }^{2,4}$. These exams should be performed on contralateral foot for comparison.
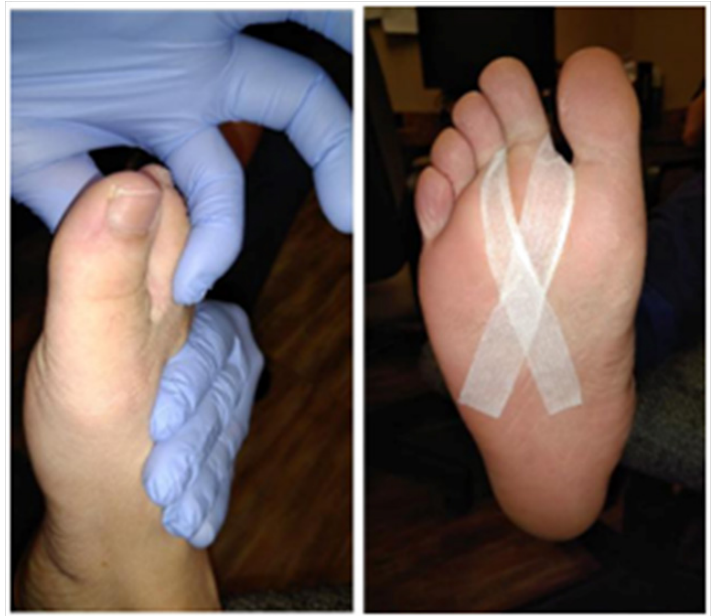

Figure 3a Demonstration of the Lachman's drawer maneuver with vertical stress applied to the proximal phalanx with one hand while stabilizing the metatarsal of the involved MTPJ with the other hand.

Figure 3b Figure 8 taping of the $2^{\text {nd }}$ MTPJ which allows the digit to be a more plantar, corrected position which helps provide pain relief.
An additional diagnostic exam is figure 8 taping of the digit (Figure 3b). With the toe in a more plantar, corrected position, patients with plantar plate dysfunction will find relief of symptoms. On weight bearing exam, attention should be directed to the distal aspect of the toe to determine if the digit purchases the ground. A high index of suspicion of plantar plate involvement should be maintained when a patient lacks ground purchase. ${ }^{9}$

Physicians must also consider concomitant pathologies leading to increased stress of the plantar plate. Commonly, patients will note a longstanding history of hallux valgus deformity which can be evaluated on radiographs. The amount of sagittal plane deformity is highlighted on the lateral view. MRI is seldom ordered because plantar plate tears are difficult to visualize due to the small size of sections of MRI images. ${ }^{9}$ Ultrasound is more sensitive than MRI to diagnose plantar plate disease, however, many physicians are not well versed in this modality and may lack the technology to perform this examination in their office. ${ }^{4}$ Physical examination remains the key to proper diagnosis of plantar plate injuries as imaging studies are not purely diagnostic. ${ }^{2}$ Plantar plate dysfunction is most often misdiagnosed as a neuroma. Possible differential diagnosis should also include ongoing arthritis, stress fractures or avascular necrosis of the metatarsal head. ${ }^{9}$

\section{Conservative treatment}

Conservative treatment depends on whether the injury is acute versus chronic. Early conservative treatment is recommended before any surgical intervention. Acute injuries can be treated with shoe modification, offloading, taping, anti-inflammatory, ice, elevation and compression. Injections are not recommended as they can mask symptoms and also weaken the plantar plate.
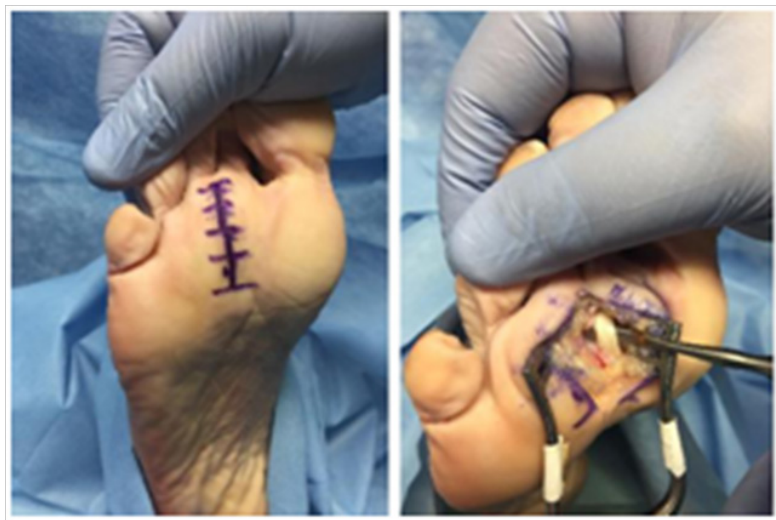

Figure 4a Linear plantar incision.

Figure 4b Plantar approach retracting the flexor digitorum longus tendon exposing the plantar plate.

\section{Plantar approach}

The plantar approach is described as making a linear incision approximately $4 \mathrm{~cm}$ in length over the MTPJ (Figure 4a). Dissection would be carried through the deep fascia down to the flexor retinaculum. Once at the retinaculum, the tendon sheath is exposed and the flexor tendon is retracted medially revealing the plantar plate underneath (Figure 4b). The tear is most commonly seen on the side of the proximal phalanx. Next, the diseased plantar plate tissue is resected. Additionally, a wedge can be resected to address any transverse plane deformity. Direct repair plantar plate is achieved with non-absorbable suture utilizing either 2-0 nylon or polypropylene. Standard skin closure is performed in layers. The patient is then placed in a surgical 
shoe and is allowed partial weight bearing on the heel. In 10-14 days, the skin sutures are removed and the patient is advanced to full weight bearing in normal shoes as tolerated. Although rare, complications for the plantar approach included a painful scar and dehiscence. This has lead to more physicians preferring the dorsal approach.

\section{Dorsal approach}

For the dorsal approach, a $3 \mathrm{~cm}$ linear incision is made either at the interspace or directly over the extensor longus tendon (Figure 5). Dissection is carried down to the extensor apparatus. A MTPJ capsulotomy is performed between extensor digitorum brevis and extensor digitorum longus. The medial and lateral collateral ligaments on the proximal phalanx are then released. Next, a Mcglamry elevator is introduced into the MTPJ to release any plantar adhesions thereby mobilizing the plantar plate.

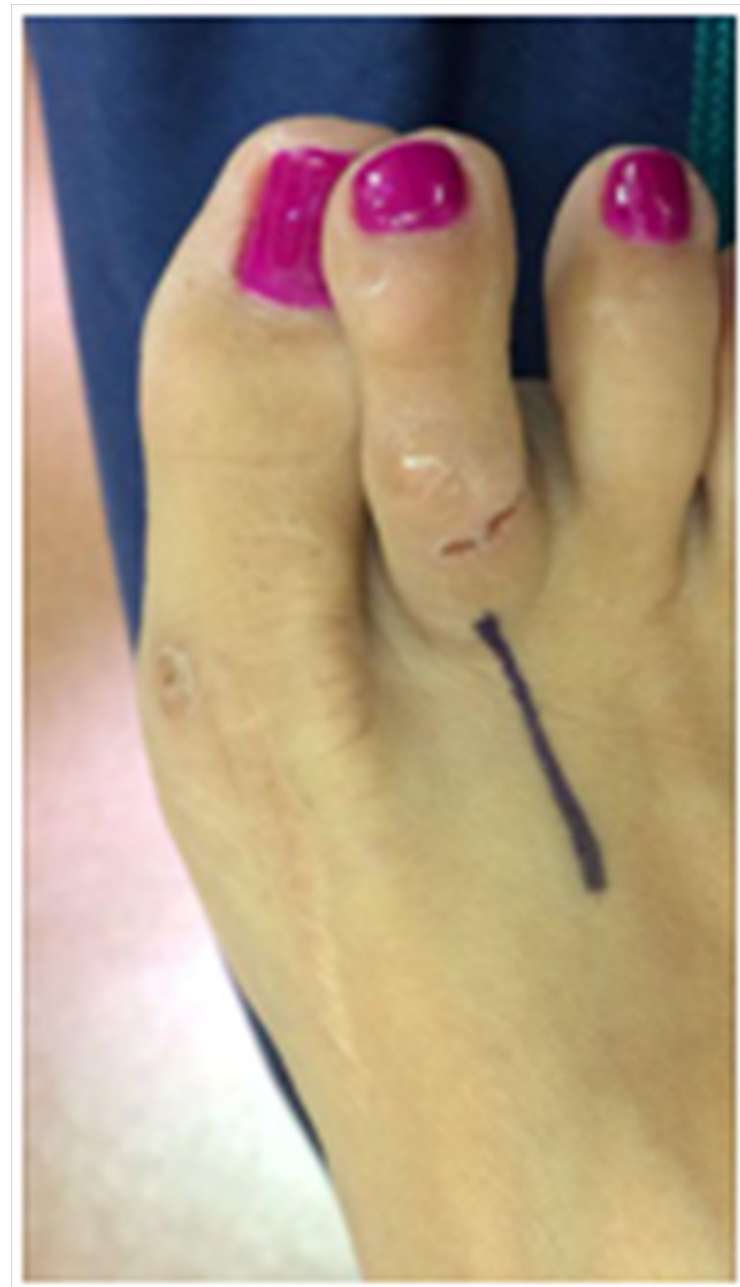

Figure $\mathbf{5}$ Incision placement for dorsal approach.

A Weil metatarsal shortening osteotomy performed beginning from dorsal articular cartilage angled at 20 degrees making sure to stay parallel to weight bearing surface. The capital fragment is translated proximally $5-10 \mathrm{~mm}$ and temporarily fixated with a k-wire. A second $\mathrm{k}$-wire is inserted into the metaphysis of the proximal phalanx so a mini joint distractor can be employed to expand the MTPJ and visualize the plantar plate. Most tears occur near the attachment of proximal phalanx. Next, carefully dissect the plantar plate off the proximal phalanx ensuring not to lacerate the flexor digitorum longus tendon which lies directly underneath.
Upon adequate visualization of the plantar plate, a full thickness flap is created to allow for a mattress suture on proximal end of the plantar plate. Next, crossed tunnels are drilled on the proximal phalanx. The suture from the proximal end is passed from plantar to dorsal through the tunnels. Next, fixate the Weil osteotomy with 2 small screws (Figure 6). The sutures are tightened with the phalanx plantarflexed 15-20 degrees and tie sutures. Standard skin closure in layers is performed. The postoperative course is identical to what is described in the plantar approach description above.

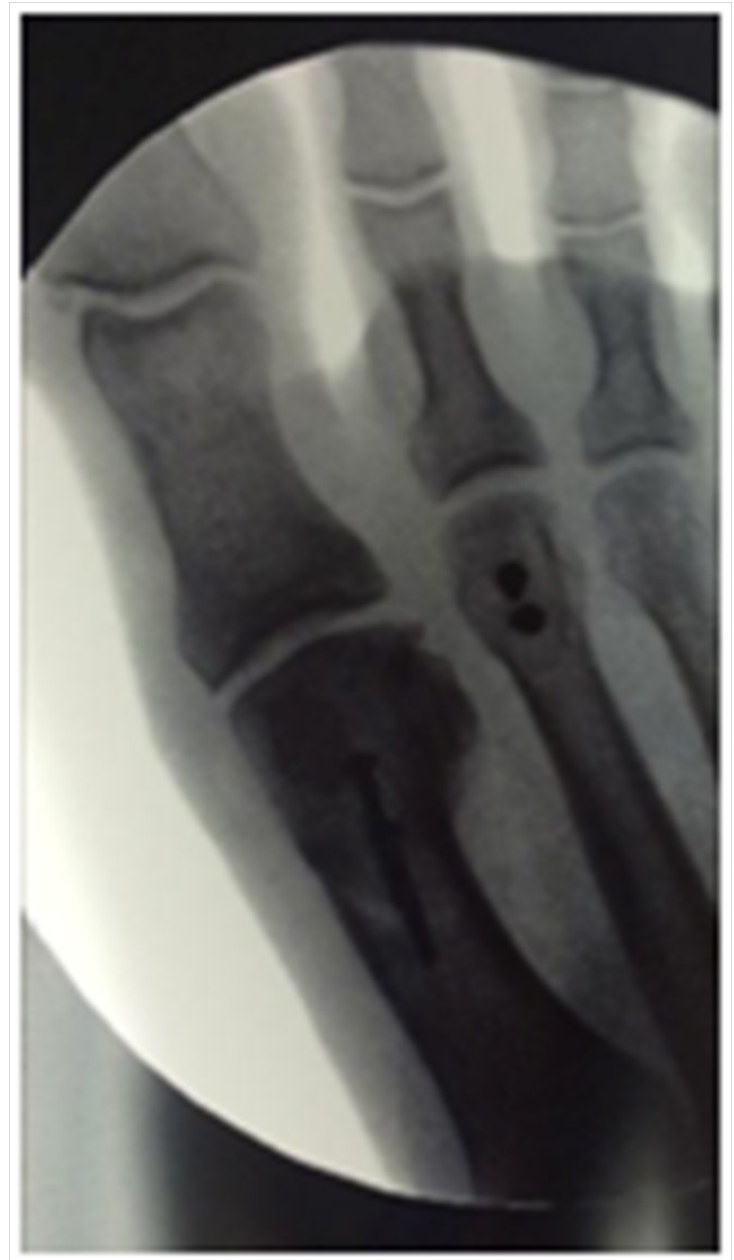

Figure 6 Weil metatarsal osteotomy with $2.0 \mathrm{~mm}$ screw fixation.

In some cases, a hammertoe deformity is also seen with a plantar plate tear. The hammertoe repair can be corrected with a proximal interphalangeal joint arthrodesis after the plantar plate repair is performed.

\section{Results/outcomes}

Overall, results for plantar plate repair are encouraging with reduction in pain and deformity. Weil et al. ${ }^{13}$ evaluated patients who underwent plantar plate repair with a Weil osteotomy and demonstrated successful results in their study population. Eighty percent of patients reported they were satisfied or very satisfied and would recommend the surgical procedure to others. Additional studies of similar surgical protocol rendered comparable results, with pain relief and resolution of deformity. ${ }^{14}$ The most common complication from the Weil osteotomy is a floating toe, which may be prevented with pinning or aggressive physical therapy following surgery. ${ }^{15}$ Complications from plantar plate repair can include but are not limited to infection, repair failure 
with recurrence, excessive shortening in osteotomies and residual toe deformity and associated pain. ${ }^{15,16}$ While there are various options for repair of plantar plate disruption, regardless of repair technique, patients generally reports less pain with improved function. ${ }^{17}$

\section{Summary}

Plantar plate pathology is recognized as a common source of lesser MTPJ deformity and multi-disciplinary research has provided a broader understanding of its pathophysiology and treatment. Surgical interventions have evolved accordingly and range from soft tissue balancing procedures to direct plantar plate repair. Numerous studies show encouraging results regarding these techniques and future investigation with long-term follow-up will fully elucidate the proper remedy for this complex condition.

\section{Acknowledgments}

None.

\section{Conflicts of interest}

None.

\section{References}

1. Mann RA, Mizel MS Monarticular nontraumatic synovitis of the metatarsophalangeal joint: a new diagnosis? Foot Ankle. 1985;6(1):18-21.

2. Kaz AJ, Coughlin MJ Crossover second toe: demographics, etiology, and radiographic assessment. Foot Ankle Int. 2007;28(12):1223-1237.

3. Siddle HJ, Hodgson RJ, Redmond AC et al. MRI identifies plantar plate pathology in the forefoot of patients with rheumatoid arthritis. Clin Rheumatol. 2012;31(4):621-629.

4. Doty JF, Coughlin MJ, Weil L, Nery C Etiology and Management of Lesser Toe Metatarsophalangeal Joint Instability. Foot and Ankle Clinics. 2014;19(3):385-405.

5. Johnston RB, Smith J, Daniels $\mathrm{T}$ The plantar plate of the lesser toes: an anatomical study in human cadavers. Foot Ankle Int. 1994;15(5):276-282.

6. Deland JT, Lee KT, Sobel M, DiCarlo EF Anatomy of the plantar plate and its attachments in the lesser metatarsal phalangeal joint. Foot Ankle Int. $1995 ; 16(8): 480-486$.
7. Barg A1, Courville XF, Nickisch F et al. Role of collateral ligaments in metatarsophalangeal stability: a cadaver study. Foot Ankle Int. 2012;33(10):877-882.

8. Bhatia D1, Myerson MS, Curtis MJ et al. Anatomical restraints to dislocation of the second metatarsophalangeal joint and assessment of repair technique. J Bone Joint Surg Am. 1994;76(9):1371-1375.

9. Camasta CA Plantar Plate Repair of the Second Metatarsophalangeal Joint. In: Southerland JT, et al. (Eds.), McGlamry's Comprehensive Textbook of Foot and Ankle Surgery. (4th edn), Lippincott Williams \& Wilkins, Philadelphia, USA, pp. 2012;187-201.

10. Yu GV, Judge MS, Hudson JR et al. Predislocation syndrome. Progressive subluxation/ dislocation of the lesser metatarsophalangeal joint. J Am Podiatr Med Assoc. 2002;92(4):182-99.

11. Louwerens JWK, Schrier JCM Rheumatoid forefoot deformity: pathophysiology, evaluation and operative treatment options. Int Orthop. 2013;37(9):1719-1729.

12. Brunet JA, Tubin S Traumatic Dislocations of the Lesser Toes. Foot Ankle Int. 1997;18(7):406-411.

13. Weil L, Weil LS, Malinoski K Anatomic Plantar Plate Repair Using the Weil Metatarsal Osteotomy Approach. Foot \& Ankle Spec. 2011;4(3):145-150.

14. Wiesel SW, Easley ME Operative techniques in foot and ankle surgery. Lippincott Williams \& Wilkins, Philadelphia, USA pp. 2011;227-232.

15. Anderson RB, Saltzman CL, Coughlin MJ Mann's Surgery of the Foot and Ankle. (9th edn), Saunders/Elsevier, Philadelphia, USA. 2014

16. Watson TS, Reid DY, Frerichs TL Dorsal Approach for Plantar Plate Repair with Weil Osteotomy: Operative Technique. Foot \& Ankle Int 2014;35(7):730-739.

17. Sanhudo AV, Gomes JLE Pull-Out Technique for Plantar Plate Repair of the Metatarsophalangeal Joint. Foot Ankle Clin N Am. 2012. 2012;17(3):417-423.

18. Nery C, Coughlin M, Baumfeld D et al. How to classify plantar plate injuries: parameters from history and physical examination. Rev Bras Ortop. 2015;50(6):720-728.

19. Siddle HJ, Hodgson RJ, Redmond AC et al. MRI identifies plantar plate pathology in the forefoot of patients with rheumatoid arthritis. Clin Rheumatol. 2012;31(4):621-629. 